\title{
Situación actual sobre la implementación del perfil básico ISO/IEC 29110 en EMP: una revisión sistemática de la literatura
}

\author{
Mirna Muñoz, Manuel Peralta \\ mirna.munoz@cimat.mx,manuel.peralta@cimat.mx \\ Centro de Investigación en Matemáticas, (CIMAT, A.C.), Unidad Zacatecas, México, Calle Lasec, \\ Andador Galileo Galilei. Andador 3, Lote 7, CP 98160, Zacatecas, México.
}

DOI: 10.17013/risti.36.1-14

\begin{abstract}
Resumen: Hoy en día, las empresas de desarrollo de software están en un contexto lleno de competitividad, así que, deben producir sistemas de software que cumplan con estándares de calidad que fomenten las buenas prácticas de desarrollo. Sin embargo, gran cantidad de estas empresas son Entidades Muy Pequeñas (EMP) con menos de 25 miembros, esto dificulta la implementación de grandes estándares como CMMI, ISO 12207, entre otros. Por esta razón, se creó el estándar ISO/IEC 29110, diseñado para este tipo de entidades que no desarrollan software crítico. En cambio, a pesar de que los procesos propuestos por ISO/IEC 29110 son ligeros, la mayoría de las EMP enfrentan barreras para su implementación. Este artículo presenta el estado actual de las EMP con respecto a la implementación del Perfil básico de ISO/IEC 29110, las herramientas de software utilizadas para respaldarlo y las debilidades que surgen dentro de estas EMP.
\end{abstract}

Palabras-clave: ISO/IEC 29110, Entidades Muy Pequeñas, implementación, barreras, herramientas de soporte.

\section{Current State about ISO/IEC 29110 basic profile implementation in VSEs: A Systematic Literature Review}

\begin{abstract}
Nowadays, software development enterprises are within a full of competitiveness context, therefore, these companies must produce software systems in compliance with quality standards that encourage good practices for development. Nevertheless, most of the software development enterprises are Very Small Entities (VSEs) with less than 25 members, this fact makes difficult to implement large standards such as CMMI, ISO 12207. For this reason, the ISO/IEC 29110 standard was created, which is specially designed for this type of entities that do not develop critical software. However, even although the processes proposed by ISO/IEC 29110 are very lightweight, most VSEs are facing barriers for their implementation. This paper aims to present the current state of VSEs regarding the implementation of the Basic Profile of ISO/IEC 29110 standard, the software tools used for supporting it, and the weaknesses that arise within these VSEs.
\end{abstract}

Keywords: ISO/IEC 29110, Very Small Entities, implementation, barriers, support tools. 


\section{Introducción}

En los últimos años, el incremento de la importancia del software como núcleo de muchas organizaciones, brinda la oportunidad para que las Entidades Muy Pequeñas (EMP) generen productos y servicios de software capaces de proporcionar soluciones de alto rendimiento a problemas de diferentes dominios (industrial, agrícola, aeronáutico, Tecnologías de la Información y la Comunicación, entre otros). Por lo tanto, es importante garantizar la calidad de sus productos de software (Clarke \& Yilmaz, 2017).

La introducción de mejoras en las EMP ayuda a implementar un conjunto de las mejores prácticas de ingeniería de software con el fin de lograr beneficios como el aumento de la calidad de su producto, reducir los tiempos de entrega, reducir los costos de producción y ampliar su cartera de clientes, entre otros (Nonoyama et al., 2016).

En este contexto, por una parte, modelos o normas como CMMI (SEI, 2010), MoProSoft (Oktaba \& Martínez, 2005) e ISO/IEC 15504 (International Standard Organization, 2004), se han desarrollado como referencia para apoyar a las organizaciones en la implementación de Mejoras en los Procesos de Software (SPI por sus siglas en inglés). Sin embargo, estos modelos se centran en proporcionar un conjunto de prácticas recomendadas que cubran "qué prácticas hacer" sin cubrir "cómo implementarlas". Además, estos modelos y estándares fueron desarrollados para las empresas más grandes sin tener a las EMP en mente (Maciaszek \& Loucopoulos, 2011).

Con respecto a esta situación, se ha desarrollado el estándar ISO/IEC 29110, que presenta un conjunto de normas y directrices que se basan en subconjuntos de elementos estándares apropiados llamados perfiles para entidades muy pequeñas (EMP). La industria del software reconoce el valor de las EMP en la contribución de productos y servicios valiosos, de acuerdo con (Muñoz et al., 2018) este tipo de organizaciones representan un promedio de 90\% en México.

Sin embargo, aunque ISO/IEC 29110 es un estándar diseñado para las EMP y adaptable a cualquier ciclo de desarrollo de software (Laporte \& O'Connor, 2016). Las EMP encuentran a algunas debilidades durante la implementación del estándar.

El objetivo de este artículo, es mostrar los resultados de una Revisión Sistemática de la Literatura (RSL) realizada con el fin de identificar el estado actual de las EMP con respecto a la implementación del Perfil Básico del estándar ISO/IEC 29110, el uso de herramientas de software para apoyarlo y las principales debilidades que aparecen dentro de estas EMP. Después de la introducción, este documento se organiza de la siguiente manera: la sección 2 presenta la RSL llevada a cabo en esta investigación, así como los resultados obtenidos después de analizar los estudios primarios identificados, y la sección 3 incluye conclusiones y trabajo futuro.

\section{Revisión Sistemática de la Literatura}

Con el fin de establecer el estado del arte con respecto a estas herramientas de software y las debilidades que surgen dentro de las EMP durante la implementación del Perfil Básico del estándar ISO/IEC 29110, se llevó a cabo una Revisión Sistemática de la Literatura. 
La Revisión Sistemática de la Literatura (RSL), es un método utilizado para identificar, evaluar e interpretar todas las evidencias disponibles, pertinentes sobre un tema o una pregunta de investigación (Kitchenham \& Charters, 2007), este protocolo se ha utilizado en otros temas de investigación como en (Hernandez et al., 2017; Mejia et al., 2016; Mejía et al., 2017; Mejia Miranda et al., n.d.; Munoz et al., 2018). RSL reduce la posibilidad de sesgo en la búsqueda de estudios, ya que es necesario definir un protocolo que especifique los métodos utilizados para guiar la Revisión Sistemática de la Literatura (Selleri Silva et al., 2015).

La RSL consta de tres fases principales: 1) Planificación de la revisión, 2) Realización de la revisión y 3) Reporte de los resultados de la revisión. Estas fases pueden parecer secuenciales, pero es importante reconocer que estas fases pueden implicar iteración. La implementación del protocolo definido para la presente investigación se describe brevemente en las secciones siguientes.

\subsection{Planificación de la Revisión Sistemática}

Ésta es la etapa inicial de la Revisión Sistemática y debe ejecutarse de la siguiente manera: confirmar la necesidad de la revisión, especificar las preguntas de investigación, listar las fuentes de datos para realizar las búsquedas y formular la cadena de búsqueda adaptándola a los motores de búsqueda seleccionados (Kitchenham \& Charters, 2007).

\section{Confirmar la necesidad para la revisión}

Para las organizaciones en el campo del desarrollo de software, es esencial implementar procesos de desarrollo basados en las mejores prácticas de modelos y estándares de calidad, sin embargo, la mayoría de las EMP en este campo no los implementan, esta situación lleva a la mayoría de sus proyectos a fallar y producir pérdidas financieras para estas organizaciones (O'Connor \& Laporte, 2012). Con la finalidad de obtener una implementación exitosa del estándar ISO/IEC 29110, las EMP necesitan un entendimiento común sobre cómo implementar los procesos adaptados a su cultura particular, y también una herramienta de apoyo específica para implementar buenas prácticas y generar evidencia a partir de estas prácticas (Lohier \& Rodríguez-Dapena, 2016), a su vez, necesitan conocer las principales debilidades que una EMP suele tener al implementar este estándar.

Es por eso por lo que esta investigación está orientada al estudio del estado actual y las principales debilidades de las EMP de desarrollo de software. Además, es importante conocer las herramientas de software que respaldan la implementación de ISO/IEC 29110, para que sea de ayuda al identificar las características que una herramienta de soporte debería cubrir para satisfacer sus necesidades.

\section{Especificar las preguntas de investigación.}

Una vez identificada la necesidad de la revisión, las preguntas de investigación se especificaron utilizando la técnica PICOC. Las preguntas de investigación se establecieron de la siguiente manera: 
RQ1.- ¿Cuál es el estado actual de las EMP de desarrollo de software con respecto al uso del perfil básico del estándar ISO/IEC 29110?

RQ2.- ¿Cuáles son las principales debilidades de las EMP al implementar el perfil básico del estándar ISO/IEC 29110?

RQ3.- ¿Qué herramientas de soporte existen para la implementación del perfil básico del estándar ISO/IEC 29110?

El objetivo de estas preguntas es obtener información sobre el estado de las EMP e identificar las necesidades de las EMP con respecto a la implementación del Perfil Básico de ISO/IEC 29110.

\section{Crear la cadena de búsqueda}

Para responder a las preguntas de investigación, se seleccionaron las palabras clave identificadas en las mismas, luego, se enumeraron sinónimos y términos relacionados con las palabras clave. Después de eso, combinando esas palabras clave y los conectores lógicos tales como "AND” y "OR" ("Y" y "O" respectivamente), se creó la cadena de búsqueda. Las palabras clave identificadas y la cadena resultante se muestran en la Tabla 1.

\section{Seleccionar las fuentes de datos}

La búsqueda de estudios primarios se realizó en bases de datos indexadas consideradas relevantes para la Ingeniería de Software, específicamente para el área de Mejora de Procesos de Software, debido al alto nivel de su uso en el campo de la investigación de ingeniería de software, estas diferentes bibliotecas digitales fueron: a) SpringerLink, b) IEEE Xplore, c) Scopus, d) Web of Science, e) ScienceDirect y f) Biblioteca digital ACM.

Es importante mencionar, que estas palabras clave, sus sinónimos o términos relacionados, y la cadena de búsqueda fueron creados en el idioma inglés, para ampliar el alcance de la investigación.

\begin{tabular}{|c|c|c|}
\hline Palabras clave & Sinónimos & Cadena de búsqueda \\
\hline \multirow{3}{*}{ Implementación } & Usage & \multirow{11}{*}{$\begin{array}{l}\text { (Implementation OR Usage OR Using OR } \\
\text { Adoption) AND (ISO/IEC 29110 OR ISO 29110) } \\
\text { AND (VSE OR VSEs OR Very Small Entities OR } \\
\text { Very Small Entity OR Very Small Enterprise) } \\
\text { AND (Tool OR Application OR Software OR } \\
\text { Support tool) }\end{array}$} \\
\hline & Using & \\
\hline & Adoption & \\
\hline ISO/IEC 29110 & ISO 29110 & \\
\hline \multirow{4}{*}{ VSE } & VSEs & \\
\hline & Very Small Entity & \\
\hline & Very Small Entities & \\
\hline & Very Small Enterprise & \\
\hline \multirow{3}{*}{ Tool } & Application & \\
\hline & Software & \\
\hline & Support tool & \\
\hline
\end{tabular}

Tabla 1 - Palabras clave y Cadena de Búsqueda Resultante. 


\subsection{Ejecución la Revisión}

La segunda fase de la RSL es la ejecución de la revisión, que se centra en la obtención de los estudios primarios. (Kitchenham \& Charters, 2007). A continuación, se muestra el proceso de selección de estudios primarios y el número de estudios encontrados en cada paso del proceso. Esta fase incluye las siguientes actividades: establecer los criterios de inclusión y exclusión, establecer los criterios de calidad de los estudios, seleccionar los estudios primarios y, finalmente, extraer datos de los estudios primarios.

Establecer los criterios de inclusión y exclusión

Para comenzar con el proceso de selección de los estudios primarios, se establecieron los siguientes criterios de inclusión y exclusión:

Criterios de Inclusión:

1. Estudios escritos en inglés o español.

2. Estudios dentro del periodo 2015-2018.

3. Estudios que describen la situación de las EMP que implementan el estándar ISO/IEC 29110.

4. Estudios que hacen referencia al uso de herramientas de soporte para los procesos de las EMP o las debilidades de las EMP.

Criterios de Exclusión:

1. Estudios que aparecen en más de una fuente.

2. Estudios que no contienen información relevante sobre la implementación del estándar ISO/IEC 29110 en las EMP.

Establecer los criterios de calidad en los estudios.

Para garantizar que la información contenida en cada estudio primario sea relevante y valiosa para la presente investigación, se establecieron dos criterios de calidad en forma de las siguientes preguntas:

SQA1.- ¿El estudio describe las debilidades en al menos un estudio de caso sobre la implementación de ISO/IEC 29110 en una EMP?

SQA2.- ¿El estudio hace referencia al uso de al menos una herramienta de soporte para implementar el estándar ISO/IEC 29110?

La evaluación de los estudios basada en los criterios de calidad se llevó a cabo seleccionando aquellos estudios que cumplían al menos una de las dos preguntas.

Selección de estudios primarios

Esta actividad sugiere definir un proceso de selección de estudios para obtener los estudios primarios para esta investigación. El proceso para seleccionar estudios primarios consistió en seis pasos:

1. Usar y adaptar la cadena de búsqueda a los motores de búsqueda seleccionados.

2. Filtrar los estudios aplicando los dos primeros criterios de inclusión. 
3. Leer los títulos y resúmenes de los estudios para identificar posibles estudios primarios.

4. Aplicar los criterios restantes de inclusión y exclusión al leer la introducción y la conclusión.

5. Aplicar criterios de calidad leyendo todo el estudio.

6. Seleccionar estudios primarios.

La Figura 1 muestra la implementación del proceso de selección de estudios descrito anteriormente. Como muestra la figura, se encontraron un total de 299 estudios durante la ejecución de la investigación en las fuentes de datos seleccionadas, pero solo 8 estudios cumplieron con los criterios de calidad, criterios de inclusión y exclusión y se consideraron como estudios primarios.
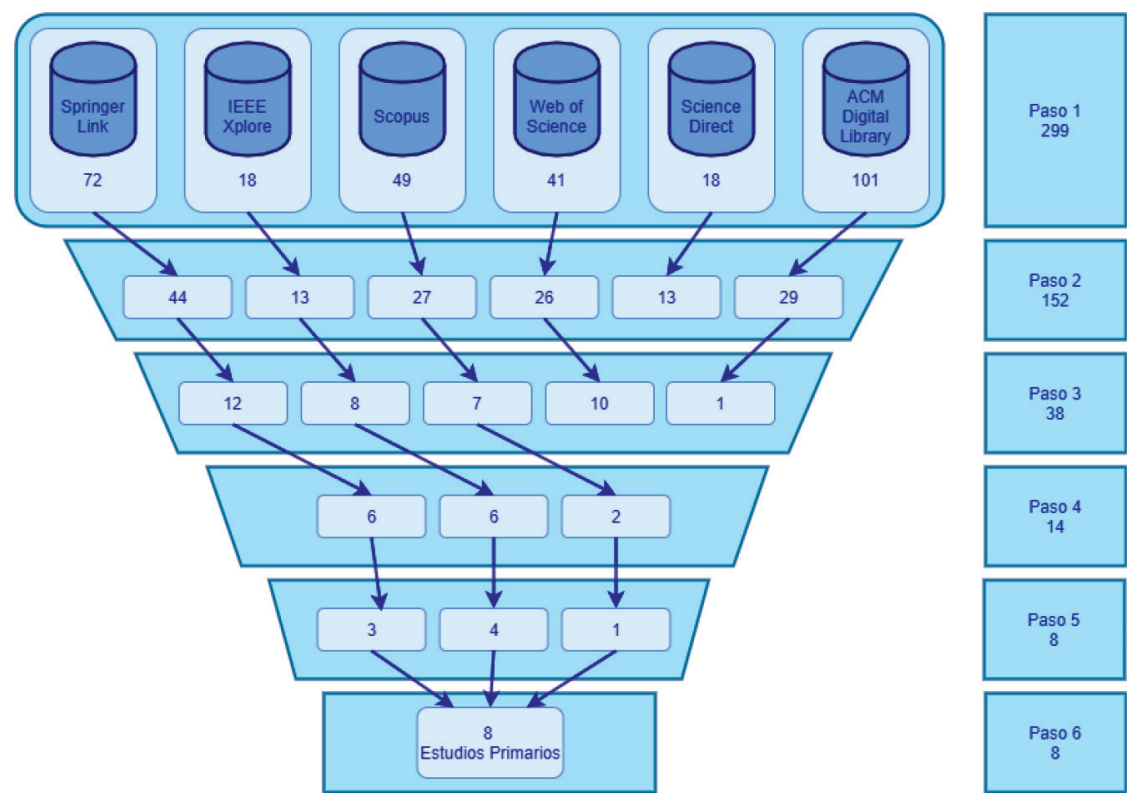

Figura 1 - Proceso de selección de estudios y resultados finales.

\section{Extracción de información}

Antes de realizar la extracción de datos, los estudios primarios se organizaron en la herramienta de gestión de archivos PDF Mendeley ${ }^{\circledR}$, estos estudios se leyeron y analizaron. Posteriormente, la información obtenida de los estudios primarios se registró en una plantilla desarrollada en la herramienta Hojas de Cálculo de Google ${ }^{\circledR}$, se diseñó un formato para la extracción de datos con las siguientes columnas: identificador, título, autores, año, palabras clave, fuente de datos, objetivo, problema, número de estudios de caso, principales debilidades, resultados obtenidos de la certificación y herramientas de soporte. 


\subsection{Resultados de la Revisión Sistemática}

En esta sección, se muestran los principales resultados obtenidos de la RSL, estos resultados proporcionan el escenario que enfrentan las EMP con respecto a la implementación del estándar ISO/IEC 29110. La Tabla 2, muestra la cobertura de cada estudio primario relacionado con preguntas de investigación y los estudios de caso contenidos en el estudio primario. Los resultados se organizan en las siguientes subsecciones y los estudios primarios se enumeran en el Apéndice A.

\begin{tabular}{|c|c|c|c|c|c|}
\hline \multicolumn{3}{|l|}{ Estudio Primario } & \multicolumn{3}{|c|}{ Preguntas de investigación } \\
\hline ID & $\begin{array}{l}\text { Estudios de } \\
\text { caso }\end{array}$ & $\begin{array}{l}\text { Estudios de } \\
\text { caso repetidos }\end{array}$ & RQ1 & RQ2 & RQ3 \\
\hline PSo1 & 3 & & $\mathrm{X}$ & $\mathrm{X}$ & $\mathrm{X}$ \\
\hline PSo2 & 74 & & & $\mathrm{X}$ & \\
\hline PSo3 & 4 & & $\mathrm{X}$ & $\mathrm{X}$ & \\
\hline PSo4 & 15 & & & $\mathrm{X}$ & \\
\hline PSo5 & 9 & 3 & $\mathrm{X}$ & $\mathrm{X}$ & $\mathrm{X}$ \\
\hline PSo6 & 11 & 4 & $\mathrm{X}$ & & $\mathrm{X}$ \\
\hline PSo7 & 5 & & & $\mathrm{X}$ & $\mathrm{X}$ \\
\hline PSo8 & 4 & & $\mathrm{X}$ & $\mathrm{X}$ & \\
\hline Total & 125 & 7 & 31 & 114 & 23 \\
\hline $\begin{array}{l}\text { Total, sin los } \\
\text { estudios de caso } \\
\text { repetidos }\end{array}$ & 118 & & 24 & 111 & 20 \\
\hline
\end{tabular}

Tabla 2 - Trazabilidad entre preguntas de investigación y estudios primarios.

Es importante resaltar que durante el análisis de datos se detectaron un total de 7 estudios de casos repetidos, para obtener el conjunto de datos más claro, estos estudios de caso se eliminaron del análisis.

RQ1. ¿Cuál es el estado actual de las EMP de desarrollo de software con respecto al uso del estándar ISO/IEC 2911O?

Las EMP que están implementando el estándar ISO/IEC 29110 reconocen que tuvo un factor de impacto positivo para su productividad. La mayoría de ellos está de acuerdo con los beneficios que la implementación de este estándar brinda a sus organizaciones. Los beneficios principales y más frecuentes se muestran en la Figura 2.

Como se muestra en la Figura 2, los beneficios más comunes están relacionados con la mejora de los procesos internos de las EMP, la reducción de las actividades de retrabajo, el incremento de la satisfacción del cliente, el acceso a nuevos clientes y el aumento de la calidad de sus productos. 


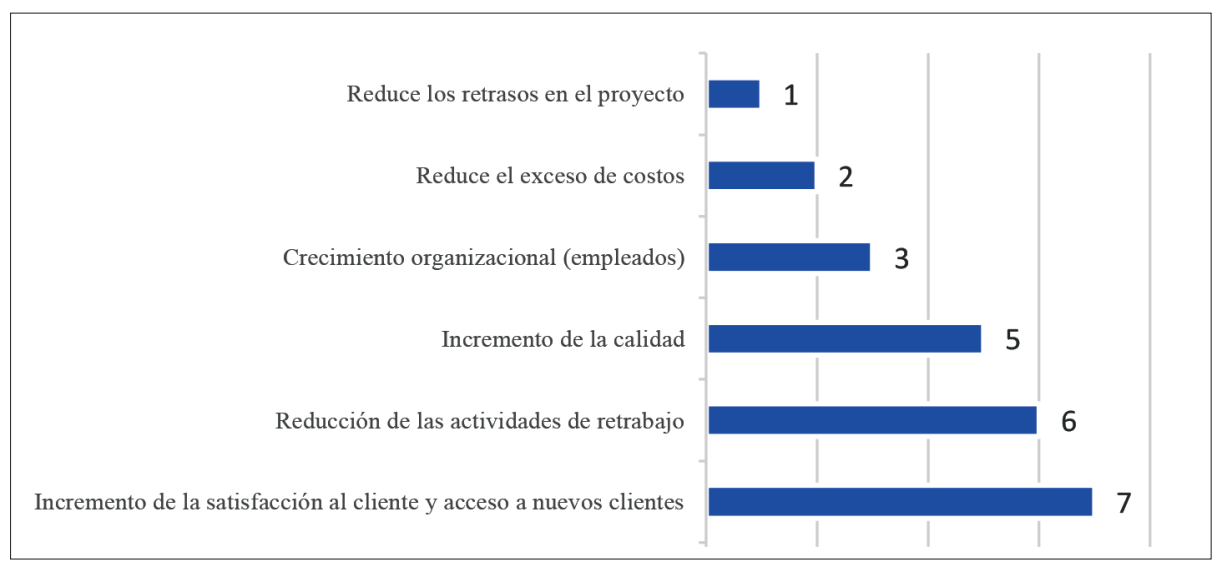

Figura 2 - Beneficios de implementar el estándar ISO/IEC 29110 (Frecuencia).

Los estudios de caso contenidos en estudios primarios como PSo1 y PSo5 informaron solo el $12 \%$ en actividades de retrabajo en contraste con el 30\% en proyectos similares (Laporte \& O’Connor, 2016). Además, el nivel de satisfacción sobre la implementación de ISO/IEC 29110 aumentó un promedio del 20\% como se muestra en PSo1.

Por otro lado, los beneficios menos comunes identificados están relacionados con la reducción de sobrecostos, el crecimiento organizacional y la reducción de demoras en los proyectos. Los estudios de caso contenidos en PSo6 reportaron un crecimiento organizacional promedio de 18 empleados. Es importante resaltar que para responder la RQ1, solo 24 estudios de caso descritos en 5 estudios primarios contenían información relevante (PSo1, PSo3, PSo5, PSo6, PSo8).

La reducción de sobrecostos y demoras en los proyectos son dos temas relacionados con la gestión del proyecto, que pueden tratarse con la ejecución de actividades correctas de monitoreo del proyecto, por lo que estos dos beneficios podrían lograrse mediante el uso de una herramienta de apoyo.

RQ2. ¿Cuáles son las principales debilidades de las EMP cuando se implementa el estándar ISO/IEC 2911O?

Dada la RQ2, siete de los ocho estudios primarios contenían información relevante sobre las debilidades de las EMP con respecto a la implementación de ISO/IEC 29110 (PSo1, PSo2, PSo3, PSo4, PSo5, PSo7, PSo8), que contenían 111 estudios de caso. La Figura 3 muestra las principales debilidades identificadas en estos estudios de caso.

La Figura 3, muestra que las debilidades más relevantes están relacionadas con: Diseño arquitectural y detallado, integración de componentes de software, aseguramiento de la calidad, verificación y validación, y casos y procedimientos de prueba. Por el contrario, las debilidades menos destacadas están relacionadas con: Proceso de gestión de proyectos, mala interpretación del estándar, falta de enfoque de mejora de procesos de software, falta de cultura de procesos organizacional y falta de capacitación adecuada de procesos. 


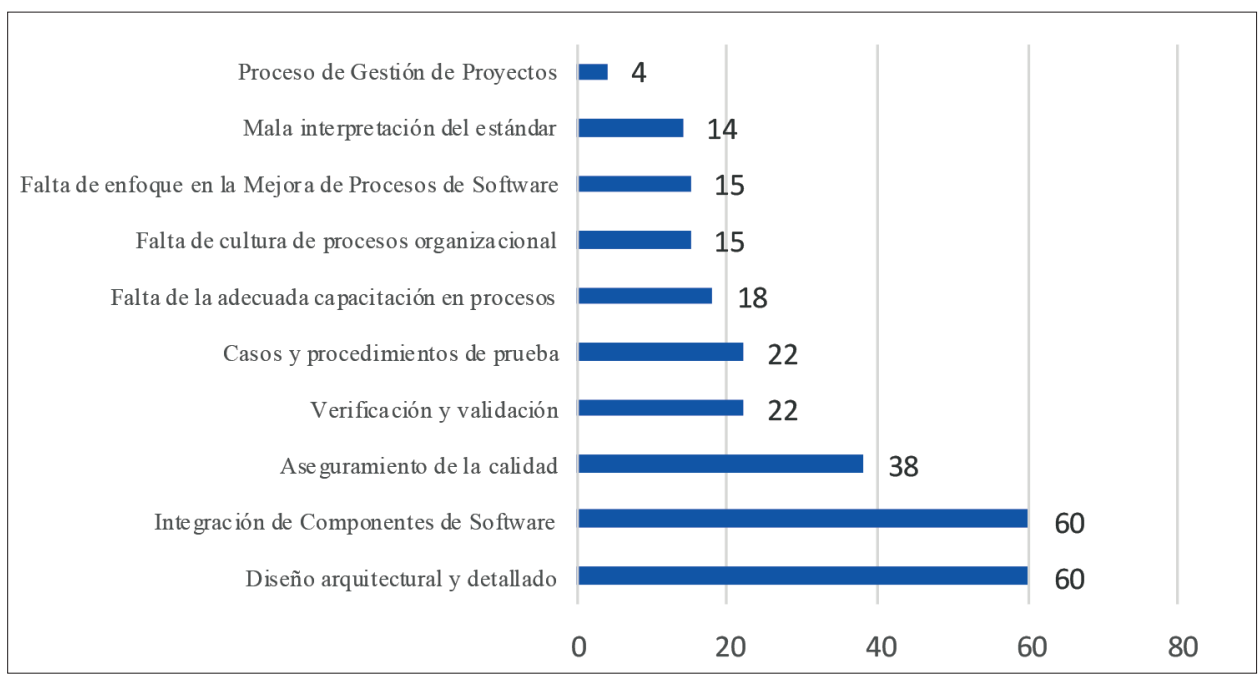

Figura 3 - Principales debilidades de las EMP con respecto a la implementación del Perfil básico de ISO/IEC 29110 (Frecuencia).

El diseño arquitectural y detallado, la integración de componentes de software y el aseguramiento de la calidad mediante verificación y validación son preocupaciones de implementación de software que, debido a las propiedades intrínsecas de estas actividades, podrían abordarse con la orientación y las recomendaciones correctas que ofrece automáticamente una herramienta de soporte.

RQ3. ¿Qué herramientas de soporte existen para la implementación del estándar ISO/ IEC 2911O?

De acuerdo con la RQ3, relacionada con las herramientas de soporte utilizadas para la implementación estándar ISO/IEC 29110, de los ocho estudios primarios, cuatro de ellos hacen referencia al uso de las herramientas de soporte (PSo1, PSo6, PSo7, PSo8) y también hacen referencia al uso de material de soporte. La Figura 4, muestra la frecuencia de uso de herramientas de soporte y material de soporte para implementar ISO/IEC 29110. Como se muestra en la Figura 4, las herramientas más utilizadas para apoyar la implementación de ISO/IEC 29110 son las herramientas internas y Bugzilla ${ }^{\circledR}$.

Una herramienta interna es un software que una EMP desarrolla para soportar su propio proceso, por lo general, este software no es para uso público y se usa para abordar solo algunas áreas de proceso específicas como se menciona en PSo1 y PSo8.

Por otro lado, los materiales de soporte más utilizados son los paquetes de despliegue (DP por sus siglas en inglés); Los elementos de un DP típico son la descripción de procesos, actividades, tareas, pasos, roles, productos, plantillas, listas de verificación, ejemplos, referencias y mapeo de estándares y modelos, y una lista de herramientas que pueden documentar el proceso. Sin embargo, hay un inconveniente para los DP, estas 
guías están destinadas a ser utilizables para cualquier EMP, sin particularizar ninguna solución específica. (Kreiner et al., 2016), y también, un DP no puede automatizar un proceso, por esta razón, este estudio solo se enfoca en herramientas de soporte.

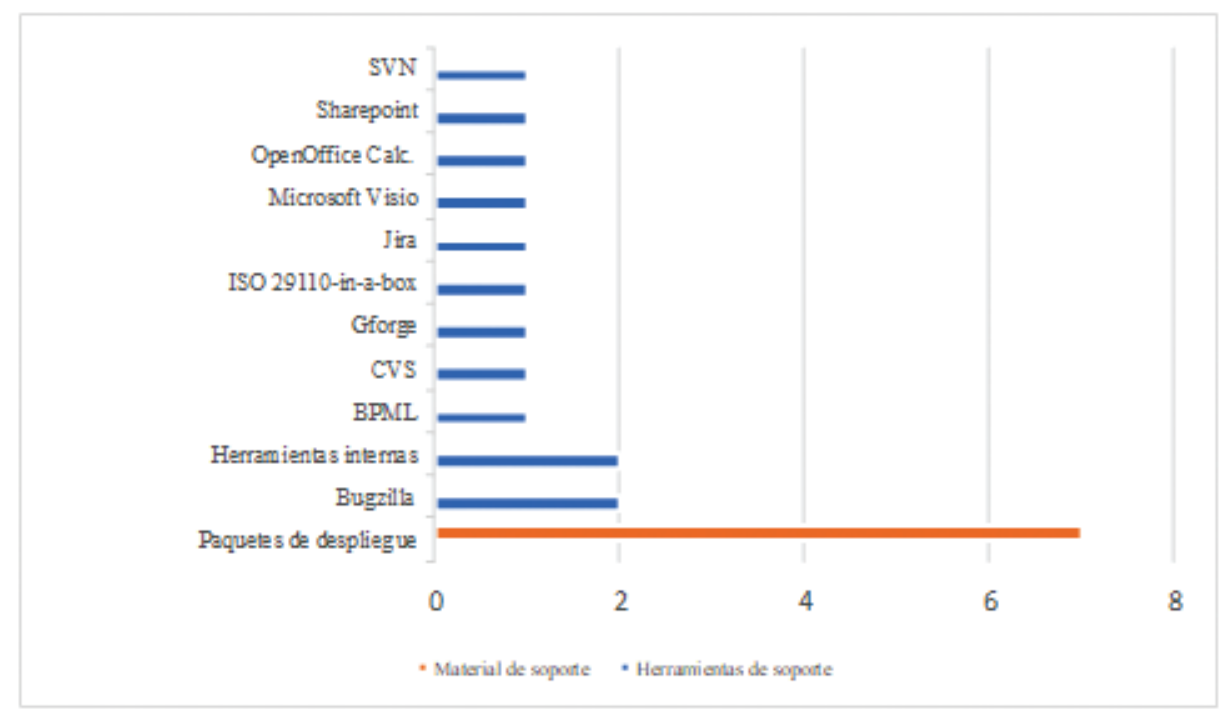

Figura 4 - Frecuencia de uso de herramientas de soporte y material de soporte.

La Tabla 3, describe el porcentaje de cobertura que tiene cada herramienta para apoyar la implementación del Perfil Básico de ISO/IEC 29110. Este porcentaje de cobertura se calculó otorgando a cada tarea del Perfil Básico ISO/IEC 29110 un peso específico, de la siguiente manera: los procesos de Gestión de Proyectos (PM) e Implementación de Software (SI) tienen el mismo peso, por lo tanto, $\mathrm{PM}=50 \%$ e SI $=50 \%$ de las tareas que debe cubrir una herramienta de soporte.

- PM tiene 26 tareas, por lo tanto, al completar una tarea de PM, una herramienta de soporte cubre un $1.9 \%$ del proceso.

- SI tiene 41 tareas, por lo tanto, al completar una tarea de SI, una herramienta de soporte cubre un $1.2 \%$ del proceso.

Tal como se muestra en la Tabla 3, las herramientas más completas para laimplementación de ISO/IEC 29110 son: ISO 29110 in-a-box ${ }^{\circledR}$, GForge ${ }^{\circledR}$ y Jira ${ }^{\circledR}$, señalando ISO 29110 ina-box ${ }^{\circledR}$ debido a que es la única herramienta cuya cobertura es del 100\%. Sin embargo, hay un inconveniente para estas 3 herramientas de soporte, todas están bajo licencia privada e implican un costo de uso, incluso los costos de uso aumentan dependiendo de la cantidad de usuarios o la cantidad de proyectos, esto aumentaría el presupuesto que las EMP deben invertir para su programa de SPI, y podría crear la percepción para las EMP de que no hay un retorno de la inversión, incluso si mejoran sus procesos (Rory et al., 2015). 


\begin{tabular}{|c|c|c|c|c|c|c|c|c|c|c|c|}
\hline $\begin{array}{l}\text { Herramienta } \\
\text { de soporte }\end{array}$ & PM.1 & PM.2 & PM.3 & PM.4 & SI.1 & SI.2 & SI.3 & SI.4 & SI.5 & SI.6 & Cobertura \\
\hline SVN (PSo7) & 1.9 & 3.8 & & 1.9 & & & & & & & $7.6 \%$ \\
\hline $\begin{array}{l}\text { SharePoint } \\
\text { (PSo7) }\end{array}$ & 1.9 & 3.8 & & 1.9 & & & & & & & $7.6 \%$ \\
\hline $\begin{array}{l}\text { OpenOffice Calc } \\
\text { (PSo7) }\end{array}$ & 15.2 & & & & & 1.2 & 2.4 & 1.2 & 4.8 & 1.2 & $26 \%$ \\
\hline $\begin{array}{l}\text { Microsoft Visio } \\
\text { (PSo7) }\end{array}$ & 1.9 & & & & & 2.4 & & & & & $4.3 \%$ \\
\hline Jira (PSo7) & 13.3 & $5 \cdot 7$ & & & 1.2 & 1.2 & & 1.2 & 1.2 & & $23.8 \%$ \\
\hline $\begin{array}{l}\text { ISO } 29110 \text { in a } \\
\text { box (PSo7) }\end{array}$ & 28.5 & 11.4 & $5 \cdot 7$ & 3.8 & 2.4 & 8.4 & 9.6 & 8.4 & 13.2 & 7.2 & $100 \%$ \\
\hline GForge (PSo7) & 15.2 & 9.5 & & 1.9 & & 2.4 & 2.4 & 2.4 & 2.4 & 1.2 & $37.4 \%$ \\
\hline CVS (PSo7) & 1.9 & 3.8 & & 1.9 & & & & & & & $7.6 \%$ \\
\hline $\begin{array}{l}\text { Herramienta } \\
\text { interna (PSo1) }\end{array}$ & 1.9 & 3.8 & & 1.9 & & & & & & & $7.6 \%$ \\
\hline $\begin{array}{l}\text { Herramienta } \\
\text { interna (PSo8) }\end{array}$ & & 1.9 & 1.9 & & & & & & & & $3.8 \%$ \\
\hline $\begin{array}{l}\text { Bugzilla (PSo1, } \\
\text { PSo7) }\end{array}$ & & & & & & & & 2.4 & 1.2 & & $3.6 \%$ \\
\hline
\end{tabular}

Tabla 3 - Cobertura (en porcentaje) de herramientas identificadas para las actividades del perfil básico de ISO/IEC 29110.

\section{Conclusiones y trabajo futuro}

Se realizó una Revisión Sistemática de la Literatura para identificar el estado actual de las EMP y el uso de herramientas de soporte para implementar ISO/IEC 29110 centrado en tres elementos clave: resultados obtenidos después de la implementación, principales debilidades y herramientas de soporte. De un conjunto total de 299 estudios, solo 8 de ellos fueron seleccionados como estudios primarios para esta investigación. Los resultados más relevantes indican que la implementación de un programa de Mejora de Procesos de Software (SPI), específicamente sobre el estándar ISO/IEC 29110, brinda beneficios y oportunidades para el crecimiento de las EMP y, por lo tanto, un incremento en la calidad de sus productos de software, manteniendo la competitividad en el mercado y mejorando las relaciones con sus clientes.

Con la ejecución de la RSL también se identificaron las principales debilidades de las EMP cuando implementan ISO/IEC 29110, las debilidades más relevantes están relacionadas con: Diseño arquitectural y detallado, integración de componentes de software, aseguramiento de la calidad, verificación y validación, y casos y procedimientos de prueba, entre otros.

Otros resultados obtenidos de la RSL indican que la mayoría de estas EMP solo usan los Paquetes de Despliegue como material de soporte. En cambio, solo unas pocas EMP utilizan herramientas de soporte para facilitar la implementación de ISO/IEC 29110. Las 
herramientas más completas para la implementación de ISO/IEC 29110 son ISO 29110 in-a-box ${ }^{\circledR}$, GForge $^{\circledR}$ y Jira $^{\circledR}$, pero solamente ISO 29110 in-a-box es la única herramienta que tiene una cobertura completa para una implementación correcta. Sin embargo, una gran desventaja para estas 3 herramientas de soporte es que todas están bajo licencia privada e implican un costo de uso que no es asequible para las EMP, lo que afecta negativamente su percepción de la implementación de un programa de SPI.

Con base en los hallazgos mencionados anteriormente, como trabajo futuro, se está creando un sistema de software destinado a brindar soporte para implementar el estándar ISO/IEC 29110, a fin de cubrir las principales debilidades detectadas y alentar la visión de las EMP para mejorar la calidad de sus productos de software mientras se facilita una implementación exitosa de ISO/IEC 29110. Para las EMP esto puede ser un factor para ganar competitividad en el mercado.

\section{Referencias}

Clarke, P., \& Yilmaz, M. (2017). Current Challenges and Proposed Software Improvement Process for VSEs in Developing Countries. Computer Standards and Interfaces, 54, 117-118. DOI: https://doi.org/10.1016/j.csi.2017.03.004

Hernandez, L., Munoz, M., Mejia, J., \& Pena, A. (2017). Gamification in software engineering teamworks: A systematic literature review. In: Proceedings of 2016 International Conference on Software Process Improvement (CIMPS), pp. 1-8. Aguascalientes, Mexico: IEEE. DOI: https://doi.org/10.1109/cimps.2016.7802799

International Standard Organization (2004). ISO/IEC 15504-1:2004, Information Technology - Process assessment - Part 1: Concepts and vocabulary. 2004. DOI: https://doi.org/10.1007/s11367-011-0297-3

Kitchenham, B., \& Charters, S. (2007). Guidelines for performing Systematic Literature Reviews in Software Engineering. Engineering, 2, 1051. DOI: https://doi. org/10.1145/1134285.1134500

Kreiner, C., O’Connor, R.V, Poth, A., \& Messnarz, R. (2016). Self-assessment as a Path to Improvement and Recognition for Small Entities. In: Proceedings of 23rd European Conference on Systems, Software and Services Process Improvement (EuroSPI 2016), pp. 514-526. DOI: https://doi.org/10.1007/978-3-319-44817-6

Laporte, C. Y., \& O’Connor, R. V. (2016). A Multi-case Study Analysis of Software Process Improvement in Very Small Companies Using ISO/IEC 29110. In: Proceedings of 23rd European Conference on Systems, Software and Services Process Improvement (EuroSPI 2016), pp. 30-44. DOI: https://doi.org/10.1007/978-3-319-44817-6

Lohier, P., \& Rodríguez-Dapena, P. (2016). Self-assessment as a Path to Improvement and Recognition for Small Entities. In: Proceedings of 23rd European Conference on Systems, Software and Services Process Improvement (EuroSPI 2016), pp. 514-526. DOI: https://doi.org/10.1007/978-3-319-44817-6 
Maciaszek, L. A., \& Loucopoulos, P. (2011). Evaluation of Novel Approaches to Software Engineering: 5th International Conference, ENASE 2010 Athens, Greece, July 22-24, 2010 Revised Selected Papers. Communications in Computer and Information Science, 230, 163-177. DOI: https://doi.org/10.1007/978-3-642-23391-3

Mejía, J., Íñiguez, F., \& Muñoz, M. (2017). Data Analysis for Software Process Improvement: A Systematic Literature Review. Advances in Intelligent Systems and Computing, 3, 571. DOI: https://doi.org/10.1007/978-3-319-56541-5

Mejia, J., Muñoz, M., Rocha, Á., \& Calvo-Manzano, J. (2016). Establishing the State of the Art of Frameworks, Methods and Methodologies Focused on Lightening Software Process: A Systematic Literature Review. Advances in Intelligent Systems and Computing, 405. DOI: https://doi.org/10.1007/978-3-319-26285-7

Mejia, J., Peralta, F. A., \& Munoz, M. (2017). Next Generation Systems - Scope and Application of Intrusion Detection and Prevention Systems (IDPS) a Systematic Literature Review. In: Proceedings of 12th Iberian Conference on Information Systems and Technologies (CISTI), pp. 1-7. Lisbon: IEEE.

Muñoz, M., Laporte, C. Y., \& Mejia, J. (2018). Implementación del Estándar ISO/IEC 29110 en Centros de Desarrollo deSoftware de Universidades Mexicanas: Experiencia del Estado de Zacatecas. RISTI - Revista Ibérica de Sistemas e Tecnologias de Informação, (29), 43-54. DOI: https://doi.org/10.17013/risti.29.43-54

Munoz, M., Mejia, J., \& Lagunas, A. (2018). Implementation of the ISO/IEC 29110 standard in agile environments: A systematic literature review. In: Iberian Conference on Information Systems and Technologies, CISTI, 2018-June 1-6. DOI: https://doi.org/10.23919/CISTI.2018.8399332

Nonoyama, T., Kabaale, E., Wen, L., David, T., \& Wang, Z. (2016). Integrating Culture Awareness and Formalisation in Software Process Assessment and Improvement for Very Small Entities (VSEs) Tatsuya. In: Porceedings of 23rd European Conference on Systems, Software and Services Process Improvement (EuroSPI 2016) (Vol. 2). Springer International Publishing. DOI: https://doi.org/10.1007/978-3$319-44817-6$

O’Connor, R. V., \& Laporte, C. Y. (2012). Software project management in very small entities with ISO/IEC 29110. Communications in Computer and Information Science, 301, 330-341. DOI: https://doi.org/10.1007/978-3-642-31199-4_29

Oktaba, H., \& Martínez, A. (2005). Modelo de Procesos para la Industria de Software: MoProSoft Brain Mapping View project An Autonomic Software Architecture For Knowledge Management In Medical Diagnosis View project. DOI: https://doi.org/10.13140/2.1.2229.5043

Rory, T. R., O'Connor, R. V., \& Dorling, A. (2015). Evaluating VSEs Viewpoint and Sentiment Towards the ISO/IEC 29110 Standard: A Two Country Grounded Theory Study. Communications in Computer and Information Science, 526, 114-127. DOI: https://doi.org/10.1007/978-3-319-19860-6 
SEI (2010). CMMI $^{\circledR}$ para Desarrollo, Versión 1.3. Equipo del Producto CMMI. DOI: http://www.sei.cmu.edu

Selleri Silva, F., Soares, F. S. F., Peres, A. L., Azevedo, I. M. De, Vasconcelos, A. P. L. F., Kamei, F. K., \& Meira, S. R. D. L. (2015). Using CMMI together with agile software development: A systematic review. Information and Software Technology, 58, 20-43. DOI: https://doi.org/10.1016/j.infsof.2014.09.012

\section{Apéndice A: Lista de Estudios Primarios}

C. Y. Laporte and R. V. O’Connor, “A Multi-case Study Analysis of Software Process Improvement in Very Small Companies Using ISO/IEC 29110," 23rd Eur. Conf. Syst. Softw. Serv. Process Improv. (EuroSPI 2016), vol. 1, pp. 30-44, 2016. https://doi.org/10.1007/978-3-319-44817-6

X. Larrucea and I. Santamaria, "Towards a survival Analysis of Very Small Organisations," 23nd European Conference on Systems, Software and Services Process Improvement (EuroSPI 2016), pp. 599-609, 2016 https://doi.org/10.1007/978-3319-44817-6

M. Muñoz, J. Mejia, and C. Y. Laporte, "Reinforcing Very Small Entities Using Agile Methodologies with the ISO/IEC 29110”, pp. 865, 88-98, 2019. https://doi.org/10.1007/978-3-030-01171-O

N. Wongsai, V. Siddoo and R. Wetprasit, "Factors of influence in software process improvement: An ISO/IEC 29110 for very-small entities," Proceedings - 2015 7th International Conference on Information Technology and Electrical Engineering: Envisioning the Trend of Computer, Information and Engineering, ICITEE 2015, 12-17. 2015. https://doi.org/10.1109/ICITEED.2015.7408904

C. Y. Laporte and R. V. O'Connor, "Implementing process improvement in very small enterprises with ISO/IEC 29110: A multiple case study analysis. Proceedings - 2016 1oth International Conference on the Quality of Information and Communications Technology, QUATIC 2016, 125-130. 2017. https://doi.org/10.1109/ QUATIC.2016.033

C. Y. Laporte, M. Muñoz, J. Mejía and R. V. O’Connor, “Applying Software Engineering Standards in Very Small Entities: From Startups to Grownups," IEEE Software, 35(1), 99-103. 2017. https://doi.org/10.1109/MS.2017.4541041

M. Muñoz, J. Mejía and A. Lagunas, "Implementation of the ISO/IEC 29110 standard in agile environments: A systematic literature review," Iberian Conference on Information Systems and Technologies, CISTI, 2018-June 1-6. 2018. https://doi. org/10.23919/CISTI.2018.8399332

M. Muñoz, C. Y. Laporte and J. Mejía, "Implementation of ISO/IEC 29110 in Software Development Centers from Mexican Universities: An experience of the Zacatecas State," RISTI - Revista Ibérica de Sistemas e Tecnologias de Informação, (29), 43-54. 2019. https://doi.org/10.17013/risti.29.43-54 